RHIC-AP-17

Booster rf Program for Heavy Ions

A. G. Ruggiero and G. Young

BNL

$8 / 22 / 85$ 
BOOSTER RF PROGRAM FOR HEAVY IONS

A. G. Ruggiero and G. Young BNL

Aug. 22, 1985

In this note we present a scenario for an $\mathrm{h}=1$ rf system to accelerate heavy ions in the Booster. Relevant Booster parameters are

$$
\begin{aligned}
2 \pi R & =201.75 \mathrm{~m} \\
\gamma_{T} & =4.5
\end{aligned}
$$

and for the beam of gold, the heaviest of the species considered,

$$
\begin{aligned}
& q=33 \text { (partially stripped ions) } \\
& A=197 \\
& N=2 \times 10^{9} \text { (total number of particles) }
\end{aligned}
$$

and also

$$
\begin{aligned}
\beta & =0.0478 \text { at injection } \\
& =0.6684 \text { at top energy }
\end{aligned}
$$

Our considerations are based on two equations:

i. The rate of acceleration

ii. The ratio of the rf bucket area to the beam bunch area.

As we said, we shall assume $\mathrm{h}=1$ with one single bunch.

\section{The rate of acceleration}

The energy gain per turn per nucleon is

$$
\Delta E=\mathrm{e} \frac{\mathrm{q}}{\mathrm{A}} \nabla \sin \phi_{\mathrm{s}}
$$

where $V$ is the peak voltage and $\phi_{S}$ the synchronous phase angle of the rf waveform.

The energy gain per unit of time

$$
\dot{\mathrm{E}}=\frac{\Delta \mathrm{E} \beta \mathrm{c}}{2 \pi \mathrm{R}}=\frac{\mathrm{eq} \beta \mathrm{c}}{2 \pi \mathrm{RA}} \mathrm{V} \sin \phi_{\mathrm{s}}
$$


but

and also

$$
E=E_{o} \gamma \text { with } E_{o}=0.9315 \mathrm{GeV} / \mathrm{A}
$$

$$
\dot{\mathrm{E}}=\mathrm{E}_{\mathrm{o}} \gamma^{3} \beta \dot{\mathrm{B}}
$$

Combining (1) and (2) gives

$$
\dot{\beta}=\frac{e q c}{2 \pi R_{0} \gamma^{3}} \operatorname{Vsin} \phi_{s}
$$

This is our first important equation which relates $\dot{\beta}$ to the product $\gamma^{3} \nabla \sin \phi_{S}$ as all the other quantities are assigned. For the booster accelerating $197 \mathrm{Au}^{33^{+}}$,

$$
\dot{\beta}=2.6722 \cdot 10^{-4} \gamma-3 \mathrm{~V} \sin \phi_{S}
$$

with $V$ in volts and $\dot{\beta}$ in $\sec ^{-1}$.

Also, a useful equation which relates $\dot{\beta}$ to $\dot{B}$, the variation with time of the bending field is

$$
\dot{\mathrm{B}} \rho=3.10715 \frac{1}{\mathrm{q}} \gamma^{3} \dot{\mathrm{B}}
$$

where $\rho$ is the bending radius in meters and $B$ is measured in Tes la. For $\beta<<1$, that is non-relativistic case, $\dot{\beta}$ constant implies also $\dot{B}$ constant.

2. The rf bucket area

In general,

$$
B=\frac{16 R}{h^{2} c} \alpha\left(\phi_{s}\right)\left[\frac{h e V q E_{o} \gamma}{2 \pi|\eta| A}\right]^{1 / 2}
$$

where $\alpha\left(\phi_{S}\right)$ is a tabulated function of $\phi_{S}$ and $\eta=\frac{1}{\gamma_{T}^{2}}-\frac{1}{\gamma^{2}}$. 
i. Capture

$$
\begin{aligned}
\phi_{S} & =0^{\circ} \\
\alpha & =1 \\
V & =450 \text { Volts }
\end{aligned}
$$

which gives at the beginning

$$
B_{0}=0.1837(\mathrm{eV} \cdot \mathrm{sec}) / \mathrm{A}
$$

The ion beam is injected with RF voltage on. It is allowed to "r." fill the bucket completely. Therefore $B_{0}$ is also the bunch area.

ii. The voltage is raised adiabatically with no acceleration $\left(\phi_{S}=0\right)$ to double its original value, that is up to 900 volts.

The new bucket area is

$$
B_{a}=0.2598(\mathrm{eV} \cdot \mathrm{sec}) / \mathrm{A}
$$

and the bunch area is still given by (6). The ratio of $B_{0}$ and $B_{a}$ represents at this time a filling factor of $\frac{1}{\sqrt{2}}$.

iii. Acceleration

We propose that the programs of $\mathrm{V}$ and $\phi_{\mathrm{S}}$ for acceleration be chosen so as to preserve the original filling factor of 0.7 during the entire cycle. This criterion would minimize the total anount of rf voltage.

Inserting all the known values in (5) we then derive the second important equation.

$$
\frac{\alpha^{2}\left(\phi_{s}\right) \vee \gamma}{|n|}=924.8 \text { volts }
$$

Equations (3) and (7) are all that are needed and they are quite general. If, for example, the $\dot{\beta}$ dependence with time is assigned during the entire acceleration cycle, one then derives $\beta$ and $\gamma$, and the two equations ( 3 and 7 ) can be solved to yield $V$ and $\phi_{S}$ versus time. 
Finally the program of the bending field can be obtained from (4).

\section{Examples}

Consider the first stage $0-50 \mathrm{msec}$ where at the end $\beta=3 \beta_{\text {inj }}=0.1434$. We can take non-relativistic approximation and set $\gamma \sim 1$.

i. Constant $\dot{\beta}$ (also $\dot{B}$ )

$$
\dot{\beta}=\frac{3 \beta_{\text {inj }}-\beta_{\text {inj }}}{50 \mathrm{msec}}=1.912 \mathrm{sec}^{-1}
$$

with this, from (3) and (7) and consulting the tables for $\alpha\left(\phi_{\mathbf{s}}\right)$, we derive

$$
\begin{aligned}
\phi_{S} & =35^{\circ}, \text { constant } \\
V & =12.5 \mathrm{kV}, \text { constant }
\end{aligned}
$$

Observe this program is not realistic because both $\phi_{S}$ and $V$ have a discontinuity. The first jumps instantly from $0^{\circ}$ to $35^{\circ}$ and the latter from $0.9 \mathrm{kV}$ to $12.5 \mathrm{kV}$.

ii. Parabolic variation of $\beta$ with time - at $50 \mathrm{msec} \dot{\beta}$ is twice the value at the same instant for constant program. That is

$$
\dot{\beta}=3.824 \text { at } t=50 \mathrm{msec}
$$

whereas $\dot{\beta}=0 \mathrm{sec}^{-1}$ at the beginning. $\mathrm{V}$ and $\phi_{\mathrm{s}}$ are not constant but vary as one can derive from ( 3 and 7 ).

\begin{tabular}{ccc}
$t$ & $0 \mathrm{msec}$ & $50 \mathrm{msec}$ \\
\hline$f_{\mathrm{rf}}$ & $71.03 \mathrm{kHz}$ & $213.09 \mathrm{kHz}$ \\
$\phi_{\mathrm{s}}$ & $0^{\circ}$ & $42^{\circ}$ \\
$\mathrm{V}$ & $0.9 \mathrm{kV}$ & $21.4 \mathrm{kV}$ \\
\hline
\end{tabular}


iii. Exponential variation.

$$
\beta=\beta \text { inj } \exp (K t)
$$

with $K=21.972 \mathrm{sec}^{-1}$,

and

$$
\dot{\beta}=\left(1.0503 \mathrm{sec}^{-1} \exp (\mathrm{Kt})\right.
$$

The results are summarized in the table:

\begin{tabular}{ccc}
$t$ & $0 \mathrm{msec}$ & $50 \mathrm{msec}$ \\
\hline$\dot{\beta}$ & $1.0503 \mathrm{~s}^{-1}$ & $3.1508 \mathrm{~s}^{-1}$ \\
$\phi_{\mathrm{s}}$ & $30^{\circ}$ & $39^{\circ}$ \\
$\mathrm{V}$ & $7.89 \mathrm{kV}$ & $19.37 \mathrm{kV}$ \\
\hline
\end{tabular}

Here also there is a discontinuity for both $\phi_{S}$ and $V$ which have to jump from $0^{\circ}$ to $30^{\circ}$ for the first and from $0.9 \mathrm{kV}$ to $7.89 \mathrm{kV}$ for the latter.

Probably the best program is a combination of the parabolic ramp until $\dot{\beta}$ reaches the value for the linear ramp, followed by a linear ramp at $\dot{\beta}=1.912$ $\sec ^{-1}$, then ending the cycle with a parabolic ramp back to $\dot{\beta}=0$.

\section{General Procedure}

This is to include both equations ( 3 and 7 ) into a computer program with the goal of optimizing the best choice of $V$ and $\phi_{S}$ according to some criterion that the rf engineering experts can develop (power, flux, losses...). 